\title{
Sex Differences in the Associations of Hemodynamic Load With Left Ventricular Hypertrophy and Concentric Remodeling
}

\author{
Thais Coutinho, ${ }^{1}$ Patricia A. Pellikka, ${ }^{2}$ Kent R. Bailey, ${ }^{3}$ Stephen T. Turner, ${ }^{4}$ and Iftikhar J. Kullo ${ }^{2}$
}

\section{BACKGROUND}

Left ventricular hypertrophy (LVH) and concentric remodeling are associated with adverse cardiovascular outcomes. We hypothesized that measures of arterial load are associated with LVH and concentric remodeling, and that associations differ by sex.

\section{METHODS}

We studied 600 non-Hispanic whites (59\% women) belonging to hypertensive sibships. By integrating arterial tonometry with echocardiography, we obtained the following hemodynamic measures: aortic characteristic impedance $\left(Z_{\mathrm{c}}\right)$, proximal aortic compliance (PAC), systemic vascular resistance, augmentation index, and carotid-femoral pulse wave velocity (cfPWV). LVH and concentric remodeling were assessed by left ventricular mass indexed to body surface area (LVMI) and relative wall thickness (RWT), respectively. LVMI was log-transformed to reduce skewness. Hemodynamic measures were indexed to body size. Sex-specific multivariable linear regression analyses adjusting for confounders were performed to assess the associations of measures of arterial load with log LVMI and RWT.

Left ventricular (LV) hypertrophy (LVH) and remodeling represent adaptive mechanisms to pressure or volume overload. According to the Laplace's Law, wall tension varies directly with pressure and inversely with wall thickness. Thus, in conditions where LV systolic pressure is increased, LV mass increases and walls thicken to maintain a normal wall tension/wall stress. ${ }^{1}$ Despite being adaptive mechanisms, LVH and concentric remodeling are associated with adverse cardiovascular events., ${ }^{2,3}$ In addition, concentric remodeling, even in the absence of $\mathrm{LVH}$, is also associated with higher risk of cardiovascular events compared to normal LV geometry, ${ }^{4}$ independently of LV mass. ${ }^{5}$ Thus, identifying individuals with concentric LV geometries (measured by high relative wall thickness (RWT)) is also relevant for risk stratification.

The response of the LV to pressure overload may differ by $\operatorname{sex}, 6,7$ suggesting greater sensitivity to pressure

\section{RESULTS}

None of the hemodynamic measures were associated with LVMI in either sex, or with RWT in men. However, in women, measures of aortic stiffness and early, pulsatile hemodynamic load were independently associated with increased RWT: $\beta \pm \mathrm{SE}=0.008 \pm 0.004$ for $Z_{c}$; $0.003 \pm 0.001$ for cfPWV, and $-0.009 \pm 0.003$ for PAC ( $P \leq 0.05$ for each). Female sex was a significant effect modifier of the associations of $Z_{C^{\prime}}$ cfPWV, and PAC with RWT ( $P \leq 0.03$ for each of the interaction terms).

\section{CONCLUSIONS}

Greater $Z_{\mathrm{c}}$ and cfP WV and lower PAC are independently associated with increased RWT in women but not in men. Our findings suggest that aortic stiffness and greater early, pulsatile hemodynamic load affect left ventricular concentric remodeling in a sex-specific manner.

Keywords: arterial stiffness; blood pressure; hypertension; left ventricular hypertrophy; left ventricular mass index; left ventricular remodeling; relative wall thickness; sex differences.

doi:10.1093/ajh/hpv071

overload in women. For instance, in isolated systolic hypertension, women are more likely to develop concentric LVH. ${ }^{7}$ Moreover, subjects with concentric hypertrophy have a much higher risk of developing heart failure than those with other LV geometric patterns, ${ }^{8}$ and concentric LV geometry has been postulated as one of the potential mechanisms for the higher prevalence of heart failure with preserved ejection fraction (HFpEF) in women. ${ }^{9}$ Thus, identifying the mechanisms promoting LV remodeling in women may help us understand the female predominance of this syndrome while highlighting potential targets for early detection and treatment.

In older, predominantly hypertensive adults, we have previously shown that ventricular-arterial interactions differ by sex. ${ }^{10}$ What remains unknown is whether there are sex differences in how the LV geometry adapts in response to
Correspondence: Thais Coutinho (tcoutinho@ottawaheart.ca).

Initially submitted February 25, 2015; date of first revision March 11, 2015; accepted for publication April 16, 2015; online publication June $1,2015$.

\footnotetext{
'Department of Internal Medicine, Division of Cardiology, University of Ottawa Heart Institute, Ottawa, Ontario, Canada; ${ }^{2}$ Department of Medicine, Division of Cardiovascular Diseases, Mayo Clinic, Rochester, Minnesota, USA; ${ }^{3}$ Department of Biomedical Statistics and Informatics, Mayo Clinic, Rochester, Minnesota, USA; ${ }^{4}$ Department of Medicine, Nephrology and Hypertension, Mayo Clinic, Rochester, Minnesota, USA.

(c) American Journal of Hypertension, Ltd 2015. All rights reserved. For Permissions, please email: journals.permissions@oup.com
} 
arterial load, and what components of arterial load (steady vs. pulsatile; early vs. late) may promote LVH and concentric remodeling in men and women. To this end, we performed a comprehensive noninvasive hemodynamic evaluation in a community-based cohort enriched for hypertension, with the goal of determining which measures of hemodynamic load are associated with higher LV mass index (LVMI) and RWT in men and women.

\section{METHODS}

\section{Study participants and assessment of baseline characteristics}

The study was approved by the Mayo Clinic's Institutional Review Board and participants gave informed consent. We studied non-Hispanic whites from the Genetic Epidemiology Network of Arteriopathy study ${ }^{11}$ who belonged to hypertensive sibships. Hypertension was defined based on a prior diagnosis of hypertension and/or current treatment with antihypertensives. Between October 2009 and December 2011, 635 participants completed the study protocol and had complete data on LVMI and RWT. We excluded 2 participants with history of aortic valve replacement, 3 with more than mild aortic stenosis, 14 with atrial fibrillation, and 16 with technically inadequate data, leaving 600 participants for final analyses.

On the day of the study, participants met with the study coordinator and completed a comprehensive questionnaire. A blood specimen was collected, serum creatinine and glucose were measured by standard enzymatic methods, and glomerular filtration rate was estimated. ${ }^{12}$ Diabetes was considered present if a subject was being treated with insulin or oral agents or had a fasting glucose level $\geq 126 \mathrm{mg} /$ dl. Smoking was defined as having smoked more than 100 cigarettes in the past. Weight (in kilograms) was measured by an electronic scale, height (in meters) by a stadiometer, and body surface area (BSA) as calculated using the Gehan Method. ${ }^{13}$

\section{Noninvasive hemodynamic assessment}

A comprehensive noninvasive hemodynamic evaluation was performed at the Mayo Clinic's Echocardiography Laboratory as previously described. ${ }^{10}$ Participants withheld vasoactive medications, alcohol and caffeine for at least 12 hours prior to the study. Brachial blood pressure was obtained by using a computer-controlled sphygmomanometer 3 times, 2 minutes apart. Applanation tonometry of the brachial, radial, femoral, and carotid arteries was performed while participants were lying supine, with simultaneous electrocardiogram. The average systolic and diastolic blood pressures were used to calibrate the peak and trough of the brachial pressure waveform, respectively. Diastolic and integrated mean brachial pressures were used to calibrate carotid, radial, and femoral pressure tracings. Next, a limited 2-dimensional echocardiogram was performed to estimate aortic flow. ${ }^{14}$ A parasternal long axis view was taken to measure the left ventricular outflow tract, followed by an apical 5-chamber view to obtain pulsed Doppler of the left ventricular outflow tract (20 seconds). This was followed by repeat carotid tonometry; carotid pressure was used as a surrogate of aortic pressure. Doppler audio was digitized online throughout these acquisitions.

Analysis of pressure data was performed in conjunction with flow data as previously described, ${ }^{10,15,16}$ using a custom software program capable of analyses of the pressure and flow data obtained (NIHem, Cardiovascular Engineering, Norwood, MA). ${ }^{10}$ With this technique we estimated several measures of arterial stiffness and hemodynamic load. ${ }^{10}$ To assess aortic stiffness, we used the carotid-femoral pulse wave velocity (cfPWV), the gold-standard measure of aortic stiffness. ${ }^{17} \mathrm{cfPWV}$ was calculated as aortic transit distance (estimated by the subtraction method) ${ }^{17}$ divided by the time delay between the foot of the carotid and femoral waveforms. We also estimated proximal aortic compliance (PAC), calculated from the Bramwell-Hill equation: co2 $=V \Delta P / \rho \Delta V$, where $V$ is aortic volume, $P$ is aortic pressure, and $\rho$ is blood density. For arterial load estimation, we calculated systemic vascular resistance (SVR) to represent the steady component of load. During pressure/ flow analyses, SVR is calculated as the impedance at zero frequency $\left(Z_{0}\right)$. We chose the aortic characteristic impedance $\left(Z_{c}\right)$ as a measure of pulsatile load. $Z_{c}$ is the pulsatile analogue of a resistance term and represents the ratio of pulsatile pressure to pulsatile flow in early systole, prior to return of the reflected pressure wave. Lastly, to determine potential effects of wave reflections on LVH and remodeling, we used the augmentation index (AIx). AIx represents the contributions of wave reflections to the central pulse pressure and was calculated as augmented pressure divided by central pulse pressure. ${ }^{10,18}$ Some physiological indices may be related to body size, which is expected to differ between men and women. Thus, we scaled SVR and PAC to BSA (SVR $\times$ BSA; PAC/BSA), $Z_{c}$ and AIx to height $\left(Z_{\mathrm{c}} \times\right.$ height; AIx $\times$ height $)$. These linear indexations were chosen because their absolute allometric exponents have been shown not to differ from the unity. ${ }^{19}$

\section{Assessment of LV structure}

A 2-dimensional transthoracic echocardiography was performed to assess LV structure. LV septal and posterior wall thickness, end-systolic and diastolic diameters were measured, and LV ejection fraction was calculated according to guidelines. ${ }^{14} \mathrm{LV}$ end-diastolic volume was estimated based on the Teichholz formula. ${ }^{20} \mathrm{LV}$ mass was derived from the simplified cubed equation formula and indexed to body surface area (LVMI) to normalize heart size to body size, according to guidelines. ${ }^{14}$ RWT was calculated as the sum of LV septal and posterior wall thickness at end diastole divided by LV internal dimension at end diastole. ${ }^{14}$

\section{Statistical methods}

Continuous variables were described as mean $\pm \mathrm{SD}$; categorical variables were described as percentages of the total. To assess differences between men and women, we employed a $t$-test for continuous variables and a chi-square test for categorical variables. 
To reduce skewness, LVMI was log-transformed. Sexspecific associations of cfPWV and indexed $Z_{c}$, PAC, SVR, and AIx with log LVMI and RWT were assessed with multivariable linear regression analyses. Generalized estimating equations were used to account for familial relatedness among participants. Covariates that may influence hemodynamic load or LV structure were included: age, mean arterial pressure, heart rate, estimated glomerular filtration rate, history of hypertension, diabetes, and smoking and use of diuretics, beta-blockers, calcium channel blockers, and inhibitors of the renin-angiotensin-aldosterone system. To determine whether sex modified the associations of measures of hemodynamic load with LVMI and RWT, we included interaction terms for sex and each load measure in the models. To reduce the possibility of type I errors, we applied the procedure described by Benjamini and Hochberg, ${ }^{21}$ using a threshold of $P=0.05$ for significance in the main effect analyses and $P=0.10$ for the interaction term analyses. Statistical analyses were performed with SPSS version 22 (IBM Corp., Armonk, NY).

\section{RESULTS}

Participant characteristics are summarized in Table 1. LVMI was higher in men than women, but RWT was similar between sexes. $Z_{\mathrm{c}}$ and AIx (absolute and indexed values) were higher in women, consistent with greater pulsatile arterial load and wave reflections in women. Results of univariate linear regression are depicted in Figures 1 (LVMI) and 2 (RWT). Arterial hemodynamic measures accounted for a small percentage of the variability in LVMI and RWT. Higher $Z_{c}$, cfPWV, and lower PAC were associated with higher LVMI and RWT in women, but not in men. In addition, higher SVR was also significantly associated with higher RWT in women only.

Independent predictors of higher LVMI and RWT are depicted in Table 2. Sex-specific associations of hemodynamic measures with LVMI and RWT are outlined in Table 3. None of the hemodynamic measures was independently associated with LVMI in men or women. However, greater cfPWV and lower PAC were independently associated with higher RWT in women, but not in men. The association of $Z_{c}$ with RWT in women was of borderline significance after applying the Benjamini-Hochberg procedure $(P=0.05)$. Neither SVR nor AIx was associated with RWT in men or women. When we used the global reflection coefficient (i.e., reflected-to-forward pressure wave amplitude ratio) instead of AIx as a measure of wave reflection, inferences remained unchanged (analyses not shown). There were significant interactions of $\operatorname{cfPWV}(P=0.02)$, indexed PAC $(P=0.02)$, and $Z_{c}(P=0.03)$ with female sex in the prediction of RWT, confirming that sex modifies the associations of aortic stiffness and pulsatile arterial load with LV concentric remodeling. The interactions of indexed SVR $(P=0.46)$ and AIx $(P=0.67)$ with sex were not significant. Of note, although men had a higher prevalence of smoking and diabetes, and were more likely to be taking antihypertensives than women, in interaction analyses, history of smoking, diabetes, and antihypertensive use did not modify the associations of arterial stiffness and pulsatile load with RWT and LVMI in men or women (analyses not shown).

\section{DISCUSSION}

In a large community-based cohort enriched for hypertension, we assessed sex differences in the associations of measures of hemodynamic load with LV structure and geometry. In women but not men, greater aortic stiffness and early, pulsatile hemodynamic load were associated with concentric LV geometry (higher RWT). To the best of our knowledge, this is the first report demonstrating sex differences in how hemodynamic load may affect LV geometry. Our findings are relevant for understanding the differential impact of arterial stiffness and hemodynamic load on LV remodeling in men and women and highlight potential pathophysiologic mechanisms linking hemodynamic load to HFpEF and adverse cardiovascular events in women.

\section{Sex differences in aortic stiffness and hemodynamic load}

We have previously shown that proximal aortic stiffness and pulsatile hemodynamic load are higher in hypertensive women than men. ${ }^{10}$ It has been proposed that potential sex differences in arterial hemodynamics may be driven by differences in body size. However, in the present study, we confirmed that sex differences persisted despite indexation of the hemodynamic variables to body size according to recommendations. ${ }^{19}$ Our findings add to a growing body of evidence supporting different age-related changes in conduit artery function between men and women, ${ }^{10,22-25}$ which can potentially explain the female predominance of cardiovascular diseases such as HFpEF and isolated systolic hypertension. ${ }^{26}$

\section{Sex differences in the associations of aortic stiffness and hemodynamic load with LV concentric remodeling}

The LV and systemic arteries are closely coupled in order to allow ample transfer of blood forward while minimizing energetic expenditure. ${ }^{27}$ As such, the stiffness (elastance) achieved by the contracting LV is closely matched to the arterial elastance. In situations where arterial elastance is increased (for instance, aortic stiffness), the LV must increase its systolic elastance in order to match the changes in arterial elastance and maintain optimal delivery of blood forward. This can be accomplished through increases in contractility, development of concentric remodeling, and increases in passive myocardial stiffness. ${ }^{27}$ Thus, LV concentric remodeling is thought to be an adaptive response to chronic increases in arterial load. Since $\mathrm{we}^{10}$ and other $\mathrm{s}^{28}$ have shown that elderly women have greater pulsatile arterial load than men, findings from our study suggest that greater age-related increases in aortic stiffness and pulsatile arterial load in women may serve a stimulus to promote concentric remodeling over time; while in men, the development of concentric remodeling appears to be independent of arterial load.

Previous studies have evaluated the prognostic effects of LV hypertrophy and remodeling, consistently showing an 
Table 1. Participant characteristics

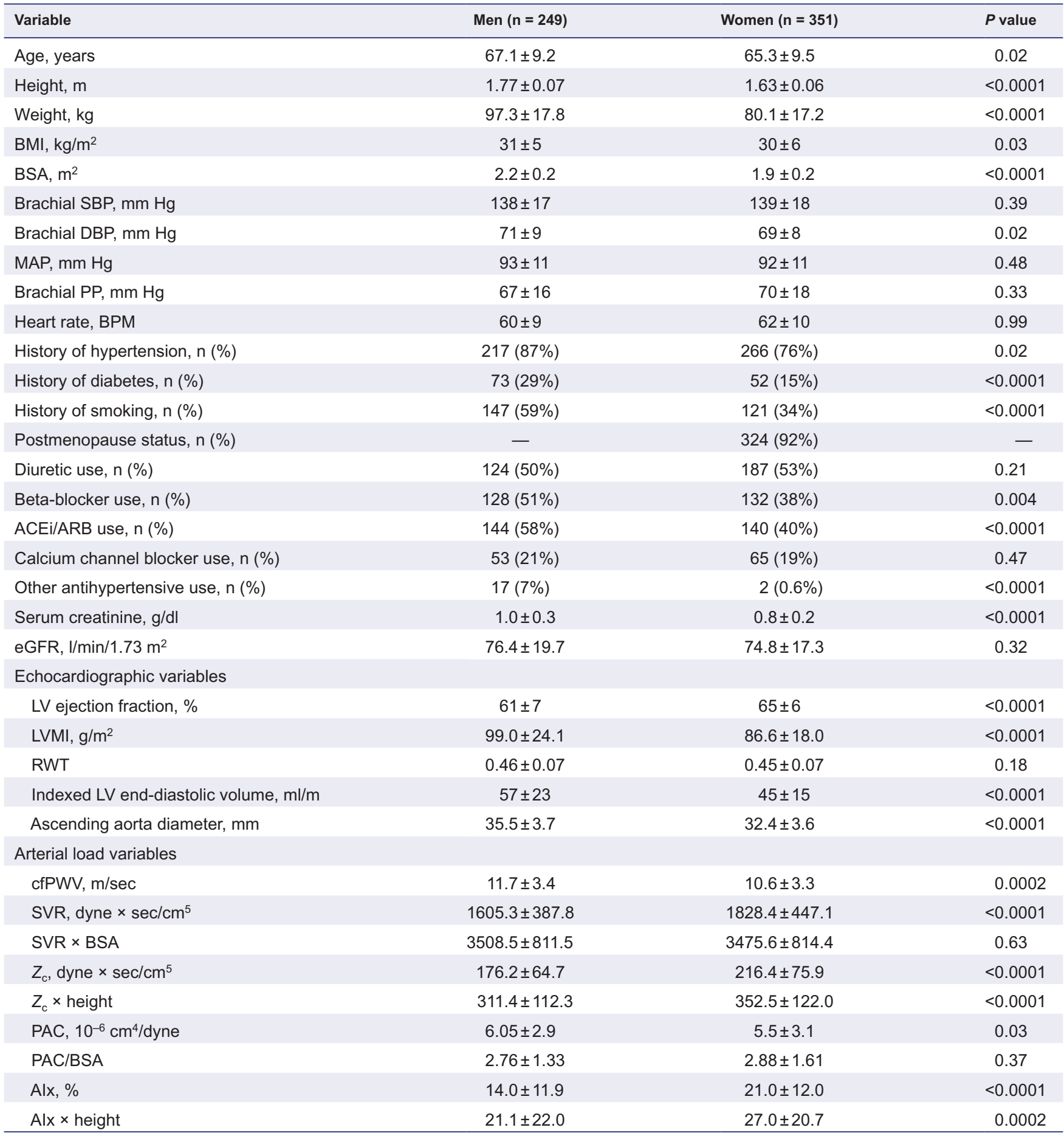

Continuous variables are presented as mean \pm SD.

Abbreviations: ACEi, angiotensin-converting enzyme inhibitor; Alx, augmentation index; ARB; angiotensin receptor blocker; BMl; body mass index; BPM, beats per minute; BSA, body surface area; cfPWV, carotid-femoral pulse wave velocity; DBP, diastolic blood pressure; eGFR, estimated glomerular filtration rate; LV, left ventricle; LVMI, left ventricular mass index; MAP, mean arterial pressure; PAC, proximal aortic compliance; PP, pulse pressure; RWT, relative wall thickness; SBP, systolic blood pressure; SVR, systemic vascular resistance; $Z_{c}$, characteristic impedance of the aorta.

association of concentric LV geometries with the greatest risk of adverse events ${ }^{2,3,8}$ and highlighting the need to better understand the pathophysiology of LV remodeling. In this context, it appears that women's hearts are more sensitive to pressure overload than men's. ${ }^{6}$ Supportive of this concept is the finding that women with isolated systolic hypertension, 

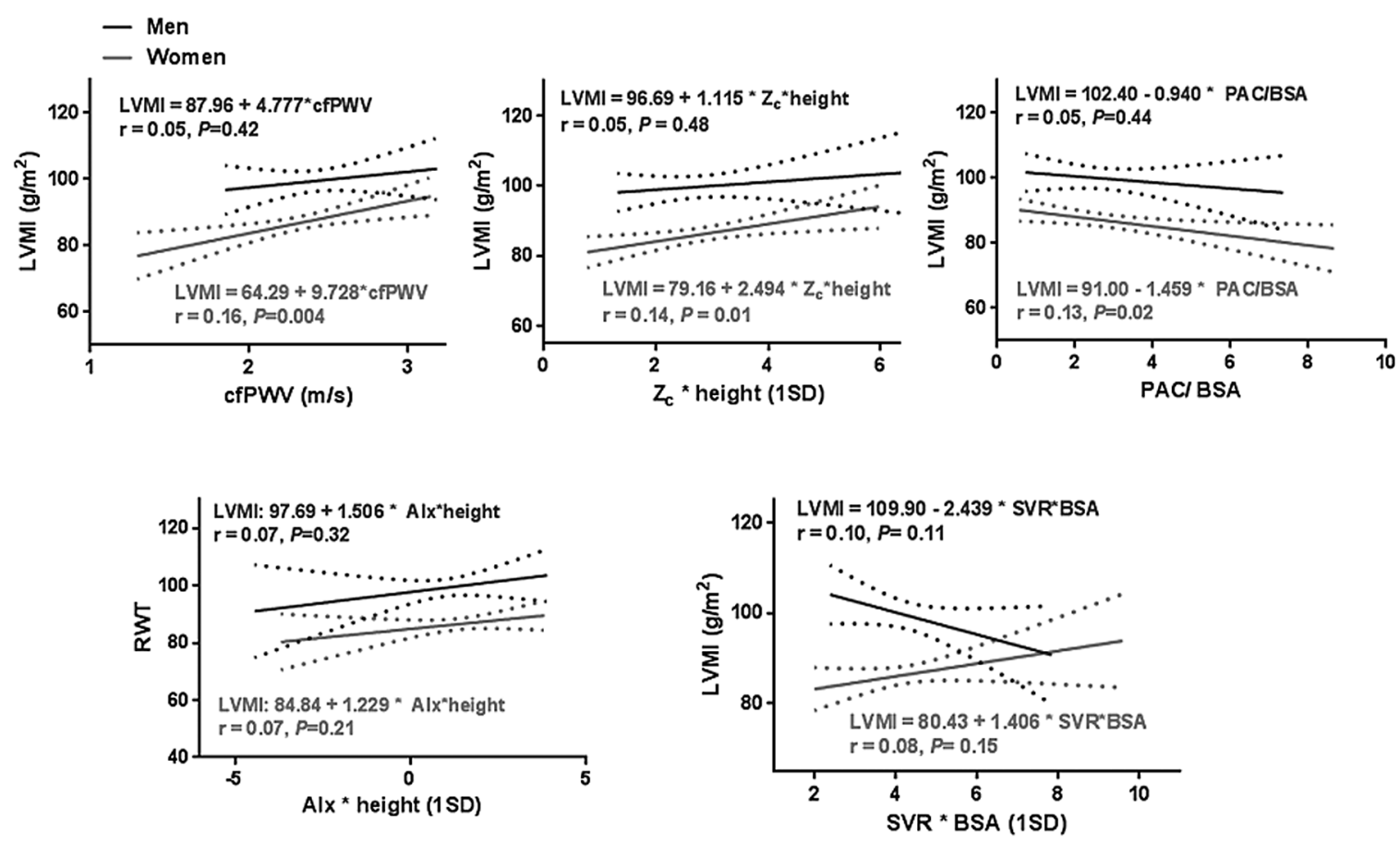

Figure 1. Sex-specific associations of measures of aortic stiffness and hemodynamic load with LVMI. In unadjusted analyses, measures of aortic stiffness and early, pulsatile arterial load ( $Z_{c}$, fPWV, and PAC) were associated with higher LVMI in women but not in men. Abbreviations: Alx, augmentation index; BSA, body surface area; CfPWV, carotid-femoral pulse wave velocity; LVMI, left ventricular mass index; PAC, proximal aortic compliance; RWT, relative wall thickness; SVR, systemic vascular resistance; $Z_{c}$, characteristic impedance of the aorta.
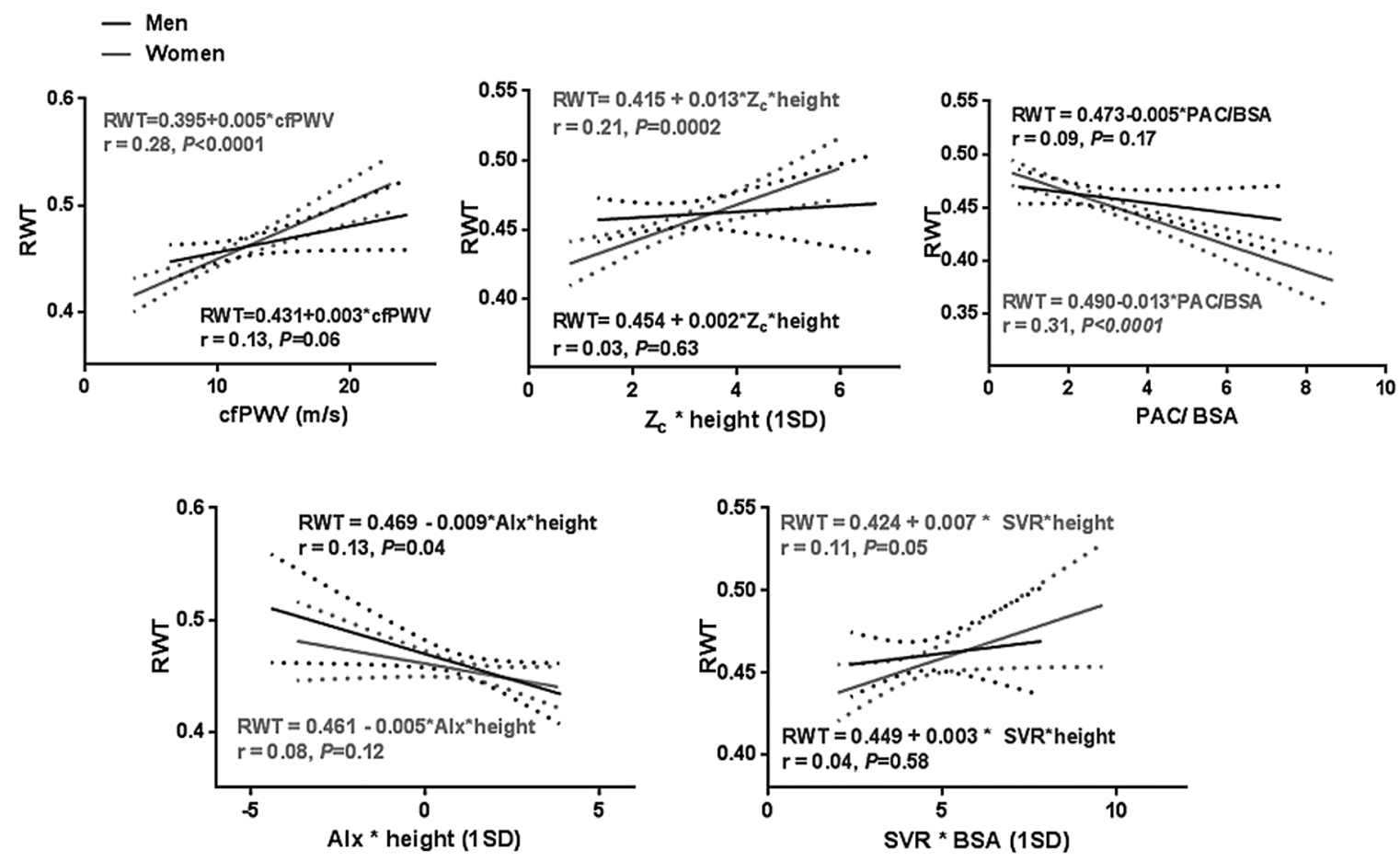

Figure 2. Sex-specific associations of measures of aortic stiffness and hemodynamic load with RWT. In unadjusted analyses, all measures of aortic stiffness and load were associated with higher RWT in women, but not in men. Abbreviations: Alx, augmentation index; BSA, body surface area; cfPWV, carotid-femoral pulse wave velocity; PAC, proximal aortic compliance; RWT, relative wall thickness; SVR, systemic vascular resistance; $Z_{c}$, characteristic impedance of the aorta. 
a condition characterized by aortic stiffness, have higher RWT than men. ${ }^{7}$ Such sex differences in LV remodeling in response to pressure overload are important when viewed in the context of HFpEF. HFpEF is more common in women, ${ }^{29}$ having hypertension and elevated RWT as common features. ${ }^{30}$ While the precise mechanisms leading to the greater prevalence of HFpEF in women remain unclear, greater LV concentric remodeling and impaired ventricular-arterial coupling in women are thought to contribute to the pathophysiology of the syndrome. ${ }^{9}$

$\mathrm{We}^{10}$ and others ${ }^{31}$ have shown that greater proximal aortic stiffness and pulsatile load are associated with impaired diastolic function ${ }^{10,31}$ and ventricular-arterial coupling ${ }^{10}$ in women, but not in men; findings that support the hypothesis that greater arterial stiffness in women may predispose to deleterious alterations in cardiovascular function and efficiency that may ultimately lead to heart failure despite a normal LV ejection fraction. The present study extends these observations by showing that greater aortic stiffness and

Table 2. Independent predictors of LVMI and RWT

\begin{tabular}{lccc}
\hline Variable & $\boldsymbol{\beta}$ & SE & $\boldsymbol{P}$ value \\
\hline log LVMI model & & & \\
$\quad$ Age, years & 0.002 & 0.001 & 0.03 \\
History of smoking & 0.044 & 0.017 & 0.009 \\
History of diabetes & 0.079 & 0.026 & 0.003 \\
\hline Heart rate, BPM & -0.005 & 0.001 & $<0.001$ \\
Calcium channel blocker use & 0.046 & 0.022 & 0.04 \\
RAASi use & 0.04 & 0.02 & 0.05 \\
RWT model & & & \\
\hline Age, years & 0.001 & 0.0003 & $<0.0001$ \\
History of hypertension & 0.028 & 0.009 & 0.001 \\
\hline History of diabetes & 0.024 & 0.009 & 0.006 \\
\hline History of smoking & 0.013 & 0.005 & 0.02 \\
\hline Heart rate, BPM & 0.001 & 0.0003 & 0.007 \\
\hline
\end{tabular}

Abbreviations: LVMI, left ventricular mass index; RAASi, reninangiotensin-aldosterone system inhibitor; RWT, relative wall thickness. pulsatile load are also independently associated with alterations in LV structure (concentric remodeling) in women only. Thus, the combination of findings points to aortic stiffness and the resulting increase in pulsatile load as potential common denominators that link hypertension, diastolic dysfunction, ventricular-arterial mismatch, and LV concentric remodeling in women, all of which are key features of patients with HFpEF. Since clinical trials to ameliorate the symptoms of HFpEF have been uniformly negative, identification of physiologic parameters that potentially predispose to HFpEF, such as arterial stiffness and measures of pulsatile hemodynamic load, will be essential in the development and testing of preventative strategies for this disease, particularly in women.

Zamani et al. ${ }^{28}$ recently evaluated associations of arterial load with LV mass and geometry in subjects from the MultiEthnic Study of Atherosclerosis (MESA). In this study, a measure of pulsatile load (total arterial compliance) was associated with greater LV mass and RWT in men and women. These findings contrast with the results from our study; however, important differences between the 2 studies were present: subjects from our study were older ( 66.0 vs. 61.3 years) and more likely to be hypertensive ( $77 \%$ vs. $42 \%)$ and white $(100 \%$ vs. $38 \%)$. Since age and the presence of hypertension are the strongest determinants of arterial stiffness, it is possible that the sex-specific effects of arterial stiffness and load on LV structure and geometry differ in the presence of hypertension. It is also possible that the impact of hemodynamic load on LV geometry may also differ based on race.

In addition, AIx, a measure of arterial wave reflections and late hemodynamic load, and SVR, a measure of steady load, were not associated with LVMI or RWT in men or women. These findings suggest that it may be the intrinsic elastic properties of the aorta and the resulting increase in early, pulsatile hemodynamic load that predominantly contribute to opposition of flow from the LV and may ultimately promote concentric remodeling of the LV myocardium in older women. These findings are in contrast with reports by Chirinos et al. ${ }^{32}$ and Borlaug et al., ${ }^{33}$ who found that late systolic loading was associated with impaired LV diastolic function. However, these studies did not assess the impact of arterial load on LV geometry and included younger individuals, in whom wave reflections are known to have a greater contribution to central pulsatile hemodynamics than in the

Table 3. Sex-specific associations of hemodynamic load with LVMI and RWT

\begin{tabular}{|c|c|c|c|c|c|c|c|c|}
\hline \multirow[b]{2}{*}{ Variable } & \multicolumn{4}{|c|}{ Log LVMI models } & \multicolumn{4}{|c|}{ RWT models } \\
\hline & $\beta \pm \mathrm{SE}$ & $P$ value & $\beta \pm \mathrm{SE}$ & $P$ value & $\beta \pm \mathrm{SE}$ & $P$ value & $\beta \pm \mathrm{SE}$ & $P$ value \\
\hline PAC/BSA & $0.010 \pm 0.012$ & 0.39 & $0.006 \pm 0.008$ & 0.42 & $0.002 \pm 0.004$ & 0.71 & $-0.009 \pm 0.003$ & 0.001 \\
\hline cfPWV (1 m/sec) & $0.001 \pm 0.005$ & 0.84 & $0.002 \pm 0.005$ & 0.61 & $0.001 \pm 0.002$ & 0.79 & $0.003 \pm 0.001$ & 0.02 \\
\hline$Z_{c} \times$ height $(1-S D$ increase) & $-0.026 \pm 0.014$ & 0.06 & $0.003 \pm 0.01$ & 0.80 & $-0.004 \pm 0.005$ & 0.46 & $0.008 \pm 0.004$ & 0.05 \\
\hline Alx (1-SD increase) & $0.005 \pm 0.023$ & 0.39 & $0.008 \pm 0.013$ & 0.51 & $-0.005 \pm 0.005$ & 0.36 & $-0.002 \pm 0.004$ & 0.60 \\
\hline
\end{tabular}

Abbreviations: Alx, augmentation index; BSA, body surface area; cfPWV, carotid-femoral pulse wave velocity; LVMI, left ventricular mass index; PAC, proximal aortic compliance; RWT, relative wall thickness; SVR, systemic vascular resistance; $Z_{c}$, characteristic impedance of the aorta. 
elderly. ${ }^{18}$ On the other hand, in the aforementioned MESA study, the amplitude of the reflected pressure wave (representing late load) was inversely associated with LV mass in older men and women and with RWT in women. Thus, it is possible that the individual effects of aortic compliance, arterial wave reflections, and loading sequence to LV structure and function may differ with aging.

Strengths of our study include the comprehensive hemodynamic evaluation and the community-based nature of our cohort. Limitations include the lack of ethnic diversity in our study cohort. In addition, almost all of the female participants from our study were postmenopausal, and it is possible that these associations may be different in younger subjects. Lastly, the cross-sectional nature of our study only provides a snapshot of complex and longstanding physiological processes that promote LV remodeling in humans. Thus, our study does not allow us to infer the causality or temporality of the associations found or to address other potential mediators of the associations of arterial load with ventricular remodeling in men and women.

In conclusion, in a community-based cohort enriched for hypertension, measures of aortic stiffness and pulsatile hemodynamic load were independently associated with higher LV RWT in postmenopausal women but not in men, corroborating the notion that women's hearts may be more sensitive to changes in aortic stiffness and highlighting the potential role of greater pulsatile hemodynamic load in the development of concentric LV geometries in women. Since concentric LV remodeling/hypertrophy is a major risk factor for mortality and cardiovascular events, and is commonly present in patients with HFpEF, our findings motivate further prospective studies aimed at assessing the role of arterial stiffness and pulsatile arterial load as precursors of LV concentric hypertrophy and as a potential therapeutic target aimed at preventing LV remodeling and its clinical consequences, particularly in women.

\section{ACKNOWLEDGMENT}

This work was supported by grant HL89354 from the National Institutes of Health.

\section{DISCLOSURE}

The authors declared no conflict of interest.

\section{REFERENCES}

1. Gaasch WH, Zile MR. Left ventricular structural remodeling in health and disease: with special emphasis on volume, mass, and geometry. $J$ Am Coll Cardiol 2011; 58:1733-1740.

2. Krumholz HM, Larson M, Levy D. Prognosis of left ventricular geometric patterns in the Framingham Heart Study. J Am Coll Cardiol $1995 ; 25: 879-884$.

3. Gerdts E, Cramariuc D, de Simone G, Wachtell K, Dahlöf B, Devereux $\mathrm{RB}$. Impact of left ventricular geometry on prognosis in hypertensive patients with left ventricular hypertrophy (the LIFE study). Eur J Echocardiogr 2008; 9:809-815.
4. Pierdomenico SD, Di Nicola M, Pierdomenico AM, Lapenna D, Cuccurullo F. Cardiovascular risk in subjects with left ventricular concentric remodeling at baseline examination: a meta-analysis. J Hum Hypertens 2011; 25:585-591.

5. Muiesan ML, Salvetti M, Monteduro C, Bonzi B, Paini A, Viola S, Poisa P, Rizzoni D, Castellano M, Agabiti-Rosei E. Left ventricular concentric geometry during treatment adversely affects cardiovascular prognosis in hypertensive patients. Hypertension 2004; 43:731-738.

6. Kostkiewicz M, Tracz W, Olszowska M, Podolec P, Drop D. Left ventricular geometry and function in patients with aortic stenosis: gender differences. Int J Cardiol 1999; 71:57-61.

7. Krumholz HM, Larson M, Levy D. Sex differences in cardiac adaptation to isolated systolic hypertension. Am J Cardiol 1993; 72:310-313.

8. Verma A, Meris A, Skali H, Ghali JK, Arnold JM, Bourgoun M, Velazquez EJ, McMurray JJ, Kober L, Pfeffer MA, Califf RM, Solomon SD. Prognostic implications of left ventricular mass and geometry following myocardial infarction: the VALIANT (VALsartan In Acute myocardial iNfarcTion) Echocardiographic Study. JACC Cardiovasc Imaging 2008; 1:582-591.

9. Scantlebury DC, Borlaug BA. Why are women more likely than men to develop heart failure with preserved ejection fraction? Curr Opin Cardiol 2011; 26:562-568.

10. Coutinho T, Borlaug BA, Pellikka PA, Turner ST, Kullo IJ. Sex differences in arterial stiffness and ventricular-arterial interactions. J Am Coll Cardiol 2013; 61:96-103.

11. Kardia SL, Rozek LS, Krushkal J, Ferrell RE, Turner ST, Hutchinson R, Brown A, Sing CF, Boerwinkle E. Genome-wide linkage analyses for hypertension genes in two ethnically and geographically diverse populations. Am J Hypertens 2003; 16:154-157.

12. Levey AS, Bosch JP, Lewis JB, Greene T, Rogers N, Roth D. A more accurate method to estimate glomerular filtration rate from serum creatinine: a new prediction equation. Modification of Diet in Renal Disease Study Group. Ann Intern Med 1999; 130:461-470.

13. Gehan EA, George SL. Estimation of human body surface area from height and weight. Cancer Chemother Rep 1970; 54:225-235.

14. Lang RM, Bierig M, Devereux RB, Flachskampf FA, Foster E, Pellikka PA, Picard MH, Roman MJ, Seward J, Shanewise JS, Solomon SD, Spencer KT, Sutton MS, Stewart WJ; Chamber Quantification Writing Group; American Society of Echocardiography's Guidelines and Standards Committee; European Association of Echocardiography. Recommendations for chamber quantification: a report from the American Society of Echocardiography's Guidelines and Standards Committee and the Chamber Quantification Writing Group, developed in conjunction with the European Association of Echocardiography, a branch of the European Society of Cardiology. J Am Soc Echocardiogr 2005; 18:1440-1463.

15. Chirinos JA, Segers P. Noninvasive evaluation of left ventricular afterload: part 1: pressure and flow measurements and basic principles of wave conduction and reflection. Hypertension 2010; 56:555-562.

16. Chirinos JA, Segers P. Noninvasive evaluation of left ventricular afterload: part 2: arterial pressure-flow and pressure-volume relations in humans. Hypertension 2010; 56:563-570.

17. Laurent S, Cockcroft J, Van Bortel L, Boutouyrie P, Giannattasio C, Hayoz D, Pannier B, Vlachopoulos C, Wilkinson I, Struijker-Boudier $\mathrm{H}$; European Network for Non-invasive Investigation of Large Arteries. Expert consensus document on arterial stiffness: methodological issues and clinical applications. Eur Heart J 2006; 27:2588-2605.

18. Mitchell GF, Wang N, Palmisano JN, Larson MG, Hamburg NM, Vita JA, Levy D, Benjamin EJ, Vasan RS. Hemodynamic correlates of blood pressure across the adult age spectrum: noninvasive evaluation in the Framingham Heart Study. Circulation 2010; 122:1379-1386.

19. Chirinos JA, Rietzschel ER, De Buyzere ML, De Bacquer D, Gillebert TC, Gupta AK, Segers P; Asklepios investigators. Arterial load and ventricular-arterial coupling: physiologic relations with body size and effect of obesity. Hypertension 2009; 54:558-566.

20. Teichholz LE, Kreulen T, Herman MV, Gorlin R. Problems in echocardiographic volume determinations: echocardiographic-angiographic correlations in the presence of absence of asynergy. Am J Cardiol 1976; 37:7-11.

21. Y B, Y H. Controlling the false discovery rate: a practical and powerful approach to multiple testing. J R Stat Soc 1995;57:289-300.

22. Shim CY, Cho IJ, Yang WI, Kang MK, Park S, Ha JW, Jang Y, Chung N. Central aortic stiffness and its association with ascending aorta dilation 
in subjects with a bicuspid aortic valve. J Am Soc Echocardiogr 2011; 24:847-852.

23. Mitchell GF, Gudnason V, Launer LJ, Aspelund T, Harris TB. Hemodynamics of increased pulse pressure in older women in the community-based Age, Gene/Environment Susceptibility-Reykjavik Study. Hypertension 2008; 51:1123-1128.

24. Mitchell GF, Lacourcière Y, Ouellet JP, Izzo JL Jr, Neutel J, Kerwin LJ, Block AJ, Pfeffer MA. Determinants of elevated pulse pressure in middle-aged and older subjects with uncomplicated systolic hypertension: the role of proximal aortic diameter and the aortic pressure-flow relationship. Circulation 2003; 108:1592-1598.

25. Segers P, Rietzschel ER, De Buyzere ML, Vermeersch SJ, De Bacquer D, Van Bortel LM, De Backer G, Gillebert TC, Verdonck PR; Asklepios investigators. Noninvasive (input) impedance, pulse wave velocity, and wave reflection in healthy middle-aged men and women. Hypertension 2007; 49:1248-1255.

26. Coutinho T. Arterial stiffness and its clinical implications in women. Can J Cardiol 2014; 30:756-764.

27. Borlaug BA, Kass DA. Invasive hemodynamic assessment in heart failure. Heart Fail Clin 2009; 5:217-228

28. Zamani P, Bluemke DA, Jacobs DR Jr, Duprez DA, Kronmal R, Lilly SM, Ferrari VA, Townsend RR, Lima JA, Budoff M, Segers P, Hannan $\mathrm{P}$, Chirinos JA. Resistive and pulsatile arterial load as predictors of left ventricular mass and geometry: the multi-ethnic study of atherosclerosis. Hypertension 2015; 65:85-92.
29. Lee DS, Gona P, Vasan RS, Larson MG, Benjamin EJ, Wang TJ, Tu JV, Levy D. Relation of disease pathogenesis and risk factors to heart failure with preserved or reduced ejection fraction: insights from the framingham heart study of the national heart, lung, and blood institute. Circulation 2009; 119:3070-3077.

30. Melenovsky V, Borlaug BA, Rosen B, Hay I, Ferruci L, Morell CH, Lakatta EG, Najjar SS, Kass DA. Cardiovascular features of heart failure with preserved ejection fraction versus nonfailing hypertensive left ventricular hypertrophy in the urban Baltimore community: the role of atrial remodeling/dysfunction. J Am Coll Cardiol 2007; 49:198-207.

31. Shim CY, Park S, Choi D, Yang WI, Cho IJ, Choi EY, Chung N, Ha JW. Sex differences in central hemodynamics and their relationship to left ventricular diastolic function. J Am Coll Cardiol 2011; 57:1226-1233.

32. Chirinos JA, Segers P, Rietzschel ER, De Buyzere ML, Raja MW, Claessens T, De Bacquer D, St John Sutton M, Gillebert TC; Asklepios Investigators. Early and late systolic wall stress differentially relate to myocardial contraction and relaxation in middle-aged adults: the Asklepios study. Hypertension 2013; 61:296-303.

33. Borlaug BA, Melenovsky V, Redfield MM, Kessler K, Chang HJ, Abraham TP, Kass DA. Impact of arterial load and loading sequence on left ventricular tissue velocities in humans. J Am Coll Cardiol 2007; 50:1570-1577. 